\title{
Limitations of high-intensity soft X-ray laser fields for the characterisation of water chemistry: Coulomb explosion of the octamer water cluster
}

\author{
Andrea Debnarova, ${ }^{a}$ Simone Techert ${ }^{* b}$ and Stefan Schmatz ${ }^{* c}$ \\ Received 26th February 2012, Accepted 25th April 2012 \\ DOI: $10.1039 / \mathbf{c} 2 \mathrm{cp} 40598 \mathrm{a}$
}

In this work, the Coulomb explosion of the octamer water cluster has been studied employing a theoretical approach. Instead of the usual methodology that makes use of classical molecular dynamics, time-dependent density functional theory has been applied to tackle the problem. This method explicitly accounts for the laser field and thus does not impose any constraint on the interaction between the laser pulse and the cluster. We focus on the effects of energetic changes in the system under high-intensity soft X-ray laser pulses. The motions of the ions and their velocities during this process show significant differences for the three applied laser intensities $\left(10^{14}, 10^{15}\right.$ and $10^{16} \mathrm{~W} \mathrm{~cm}^{-2}$ ). Very strong soft X-ray free electron laser (FEL) pulses must be short to allow for investigations of ultra-fast wet chemistry, according to the principle of collect and destroy.

\section{Introduction}

One of the possible applications of free electron laser (FEL) science is to utilize the FEL radiation for monitoring chemical reactions in real time. In particular, soft X-ray free electron laser radiation has appropriate physical properties for the investigation of such processes in the bulk liquid phase. Soft X-ray radiation has the capability of studying redox properties of light elements and transition metal complexes and the structural modifications that are directly linked to the redox changes. A further field of investigations addresses the soft X-ray spectroscopy of bulk water. As in FEL imaging techniques one of the limiting factors for FEL spectroscopy concerns radiation damage; it is postulated that radiation damage sets a limit for the probe-pulse intensity a sample can be exposed to. At high intensities, the energy transfer from the X-ray field to the molecules or clusters in the sample becomes so dominant that on a very short time-scale due to single- and multi-photon excitations the sample transforms into a collection of highly charged ions embedded in a quasifree electron gas. The formation and the dynamics of this state is called a Coulomb explosion. Necessarily such a process influences any measurement performed on the sample in a number of ways.

\footnotetext{
${ }^{a}$ Max Planck Institute for Biophysical Chemistry, 37070 Göttingen, Germany.E-mail: adebnar@gwdg.de

${ }^{b}$ Max Planck Institute for Biophysical Chemistry, IRG Structural Dynamics of (bio)chemical Systems, 37070 Göttingen, Germany. E-mail: stecher@gwdg.de

${ }^{c}$ Institut für Physikalische Chemie, Universität Göttingen,

37077 Göttingen, Germany.E-mail: sschmat@gwdg.de
}

The process of Coulomb explosion can be generally divided into three main time-phases. In the first phase, the laser light couples to the atoms as if they were isolated, while the cluster environment does not have any effect; this phase is dominated by photo ionizations. In the second phase, the cluster expands due to the strong electrostatic repulsion between ions that were created in the first phase, together with 'inner ionized' or 'quasi free' electrons which are highly energetic particles still bound to the cluster. In the third phase, energy redistribution sets in and recombinations occur. ${ }^{1,2}$

Current intensity levels at soft X-ray FELs are close to Coulomb explosion limits. A way to avoid the problem with sample disintegration in the course of spectroscopic measurements is theoretically straightforward: the probe-pulse must be both intense and short enough to provide spectroscopic information before structural and electronic changes due to the Coulomb explosion erode the collected spectroscopic data (according to the more generalized FEL principle of collect and destroy).

In a publication by Bergh, Timneanu and van der Spoel, ${ }^{7}$ the X-ray laser-matter interaction model is expanded by accounting for the free-electron gas through iterative use of the Poisson-Boltzmann equation. This electron gas created by Auger and secondary electrons is assumed to be described by a simple Maxwell-Boltzmann distribution. The test systems in this case were relatively large water clusters containing 660 or 1320 molecules, respectively. It was shown that the presence of the electron gas slows down the Coulomb explosion. A radiationhydrodynamic model for the interaction of a soft X-ray laser pulse with matter was studied by Hau-Riege et al. ${ }^{8}$

A detailed model of laser-matter interaction can be provided by time-dependent density functional theory (TDDFT) 


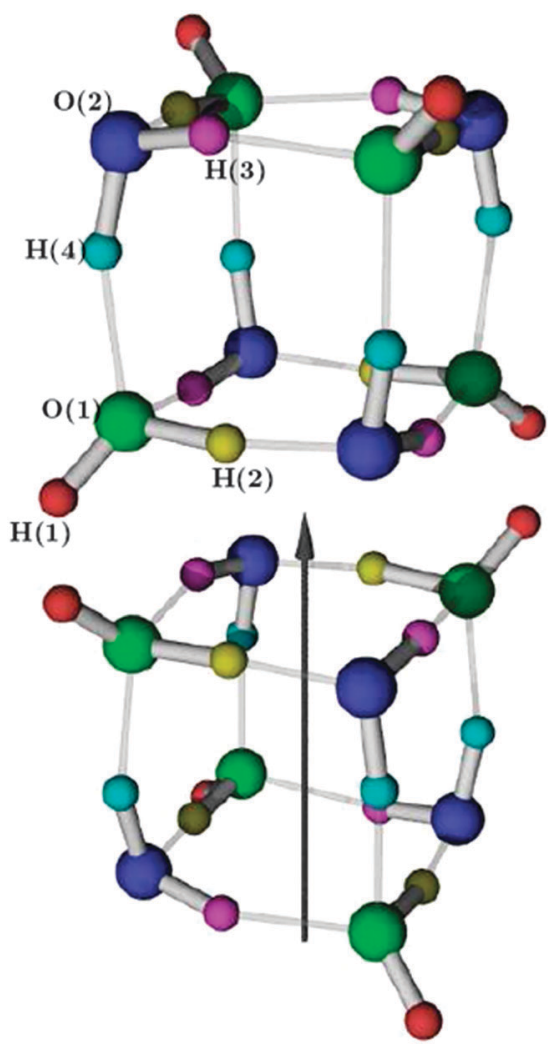

Fig. 1 Water octamer with symmetrically independent atom groups colored separately. The arrow indicates the axis of the $C_{4}$ rotation, which followed by a reflection in the perpendicular plane is the generator of the $S_{4}$ symmetry group of the cluster.

in its formulation beyond linear response. Since this quantumchemical approach is computationally expensive, the size of the studied system has to be limited accordingly. The $\left(\mathrm{H}_{2} \mathrm{O}\right)_{8}$ water cluster with $S_{4}$ symmetry was chosen as a test system (see Fig. 1) in our calculations. It is small enough to minimize the computational costs of TDDFT, while the geometry of this cluster allows for a limited level of volume effects, such as freeelectron gas shielding. This water octamer is notable because of its tri-coordinated hydrogen-bond deficient water structure. It has been an object of both theoretical ${ }^{9}$ and experimental ${ }^{10}$ studies. The two energetically lowest water octamer conformations form a cube with the water molecules positioned at the corners, oriented to form $D_{2 d}$ or $S_{4}$ symmetric structures. The tri-coordinated structures are known to form water ${ }^{11}$ and ice ${ }^{12}$ surfaces, as opposed to the tetra-coordinated water that dominates the bulk.

The dynamics of molecules under the influence of fields with very high intensities and very short pulse lengths, as from FEL fields, is by nature highly non-linear. A convenient method for describing the behavior of a system like this is TDDFT in its form beyond linear response. The full TDDFT is a promising method offering a quantum-chemical approach to the problem as opposed to the widely used molecular dynamics treatment ${ }^{3-7}$ allowing the study of small molecular systems. The coupling of the laser field to the molecular system is formulated in a semiclassical way, the so-called minimal coupling scheme, where the laser is treated as a classical electromagnetic field. In the TDDFT implementation employed in this work, the external laser field is described using the dipole approximation, which is always justified for optical wavelengths, while for hard X-rays it is satisfied only in the case of inner-shell electrons.

It should be noted that the TD density functionals used for describing the electron behavior in strong fields are only in the developmental stage. Therefore, experiments such as timedependent scattering measurements may prove invaluable for gaining a better understanding of the behavior of electronic structures in strong fields and help to improve the precision of the computational methods and the development of TD density functionals.

The theoretical treatment of the Coulomb explosion problem incorporates a number of approximations. To simplify the problem it might be assumed that the laser couples to the electrons, while the coupling to the atomic nuclei can be neglected. This approximation is used in the type of calculations where the laser-matter interaction is accounted for stochastically. TDDFT beyond linear response, however, through the explicit presence of the laser field in the Hamiltonian accounts even for this small effect that the strong laser field exerts on the nuclei. The coupling is formulated in a semiclassical way, resulting in the minimal coupling scheme.

An important question for structural measurements with strong X-ray laser pulses is how the behavior of the Coulomb explosion changes with the field strength. As the pulse lengths generally considered are in the order of 10 to 100 femtoseconds, the structural changes during this time should be small if a strong, well-defined scattering signal is to be obtained. The forming free-electron gas has a dominant effect on the structural data deterioration. TDDFT is a convenient method for the detailed description of this electron-gas formation.

The aim of the current study is to provide a computational rationalization of soft X-ray FEL-matter interaction on systems which are relevant for chemistry under real conditions. Such a case is the present octamer water system which is one of the dominant water clusters in bulk water.

In the following, we study the Coulomb explosion behavior with respect to four different field intensities for the soft X-ray regime of $120 \mathrm{eV}$. The strongest intensity studied, $10^{16} \mathrm{~W}$ $\mathrm{cm}^{-2}$, causes a very fast Coulomb explosion with significant changes in the structure even at the short computed time interval of $5 \mathrm{fs}$. For a comparison, three weaker fields with intensities $10^{13}, 10^{14}$ and $10^{15} \mathrm{~W} \mathrm{~cm}^{-2}$ are considered as well. From the point of view of spectroscopic measurements, these weaker fields show a more promising fragmentation rate, however one must not forget the relation between the field strength, the absorption and inelastic and elastic scattering signal intensity which leads to the necessity for very strong fields in the case of small samples and is particularly important for real-time chemical reaction investigations.

\section{Computational details}

For the treatment of the interaction between matter and strong laser fields within the framework of TDDFT, the time-dependent Kohn-Sham equations have to be solved explicitly. This approach has been implemented in the quantumchemistry program package OCTOPUS ${ }^{13,14}$ as briefly described 
in the following. In OCTOPUS, the atomic nuclei are treated classically according to Ehrenfest dynamics. The total system under consideration is represented as two coupled dynamical systems consisting of (a) an ensemble of non-interacting electrons obeying the Kohn-Sham equations and (b) a classically described set of atomic ions. The number of active electrons is reduced by using norm-conserving pseudopotentials to describe the core electrons. The time-dependent Kohn-Sham equations are solved by integration in real time with all quantities discretized on a grid in real space. The electromagnetic laser field is described classically as an additional external potential and the calculation is limited to the dipole approximation. For the laser field we then have

$$
E_{\text {laser }}(\boldsymbol{r}, t)=E_{0} f(t) \sin (\omega t) \hat{\boldsymbol{p}} \cdot \boldsymbol{r}
$$

with field strength $E_{0}$, frequency $\omega$, the envelope of the pulse $f(t)$ and the polarization vector $\hat{\boldsymbol{p}}$.

The OCTOPUS program package has been successfully applied. ${ }^{15,16}$ In a paper by Isla and Alonso, ${ }^{15}$ the Coulomb explosion and fragmentation of deuterium clusters $\mathrm{D}_{13}^{+}$have been studied. In a TDDFT study of the Coulomb explosion of $\mathrm{D}_{2}$ by Livshits and Baer, ${ }^{16}$ three different approaches are compared: the time-dependent Hartree-Fock method, the simplest TDDFT method ALDA (adiabatic local density approximation) and TDDFT with the Baer-Neuhauser functional. ${ }^{17}$ This work concludes that the Baer-Neuhauser functional, which does not suffer from long-range selfrepulsion while incorporating ground state correlation effects similar to ALDA, does not offer any significant improvement to the latter method.

Due to the fact that the OCTOPUS program uses a pseudo potential description for core electrons, the studied laser wavelength has to be larger than the oxygen 1s absorption edge at $4 \mathrm{~nm}$. We chose the soft X-ray wavelength of $10 \mathrm{~nm}$, corresponding to a photon energy of $120 \mathrm{eV}$, which is already reachable with the FLASH-FEL at the DESY in Hamburg. This means that in principle all 64 valence electrons which are treated in this work can be excited in the simulation. It also keeps us well within the dipole approximation considering the size of the cluster. The interactions between core electrons which are described by the pseudo potential and the laser field are not accounted for explicitly. The time step in the simulation is chosen to be 0.1 attoseconds.

In our calculations, we make use of the ALDA approach which suffers from well-known shortcomings such as selfinteraction effects, but has the advantage of being a simple, extensively studied model. We note that the problems of linear response TDDFT in describing charge transfer excitations are due to the perturbative treatment used in that approximation, ${ }^{18}$ and are not expected to appear in the present calculation, which goes beyond perturbation theory by employing an explicit time-integration.

There are important differences between hard X-ray and soft X-ray laser-matter interactions. The higher energy end of the water absorption spectrum is dominated by the two main lines - the $L$-edge at about $30 \mathrm{eV}$ and the $K$-edge at about $530 \mathrm{eV} \cdot{ }^{19-21}$ Table 1 shows a few main X-ray scattering cross sections for oxygen wherefrom it can be seen that the main
Table 1 Photoelectric, elastic and inelastic scattering cross sections of oxygen atoms at different photon energies in barns/atom, taken from ref. 21

\begin{tabular}{llll}
\hline Energy $(\mathrm{eV})$ & Photoelectric & Elastic & Inelastic \\
\hline 100 & $1.65 \times 10^{6}$ & 42.5 & $2.83 \times 10^{-3}$ \\
1000 & $1.26 \times 10^{5}$ & 39.9 & $2.29 \times 10^{-1}$ \\
10000 & $1.47 \times 10^{2}$ & 6.85 & 3.44 \\
\hline
\end{tabular}

contribution to the radiation damage at $100 \mathrm{eV}$ results from photo ionization. The dominant photoelectric cross section drops four orders of magnitude between $100 \mathrm{eV}$ and $10000 \mathrm{eV}$ having a significant impact on the decrease in the total scattering cross section for hard X-rays.

An important physical characteristic with respect to the radiation damage is the inelastic electron scattering cross section. With hard X-ray photon pulses the main contribution to electron scattering events originates from Auger electrons ${ }^{22}$ because the cross section for scattering events with high-energy photoelectrons is very small as they are expected to leave the sample very fast. In the case of soft X-ray pulses, the photoelectrons have energies in the range where the electron scattering cross section is high and thus contribute to the 'inner ionized' electron-gas formation. However, it is important to note that for strong fields these cross sections have to be modified to take multi-photon effects into account which are included in the present calculation, since the field is explicitly accounted for in the simulation (in contrast to other approaches based on perturbation theory).

The simulations of the water cluster $\left(\mathrm{H}_{2} \mathrm{O}\right)_{8}$ in a strong $\mathrm{X}$-ray field start with the water octamer in its electronic ground state structure. The initial guess for the geometry optimization of this cluster was taken from the Cambridge Cluster Database. ${ }^{23}$

In this work we are mostly interested in the quantumchemical description of the very initial processes induced by FEL pulses into the water cluster.

In order to oversample the dynamic electronic processes induced by pulsed FEL photons, the pulse width of $7 \mathrm{fs}$ consisted of time-displaced 500 as pulses. The rising time of the whole FEL pulses was simulated through very narrow Gaussian functions. Since the eletronic decay times (Auger, photoelectron etc.) are on the order of some femtoseconds, these relaxation processes are slower than incoming FEL pulse photons which leads to an accumulation of kinetic energy from this dynamical point of view.

From the technical-experimental point of view, there are various discussions of shortening FEL pulses to the attosecond time regime. Although the discussion is still hypothetic, there exist potential ways of realizing attosecond FEL pulses within a couple of years. The calculations start at 1 FWHM left of the pulse maximum which seems to be a reasonable choice according to our comprehensive test calculations. The building-up phase is not relevant for the final results. Due to this fact, our figures show data counted from the pulse maximum (i.e. data corresponding to a 'negative' time-scale are not displayed). Furthermore, only 6 fs propagation time are shown despite the fact that the actual time intervals covered up to $200 \mathrm{fs}$. We performed many calculations with 
coarser time grids and even longer time intervals, but the evidence was that 6 fs were sufficient since after $10 \mathrm{fs}$ obvioulsly no movement of the nuclei could be seen. The production of Auger electrons takes place within the first 6 fs.

In order to achieve convergence of the computed properties, we had to define the size of the spatial box, while the parameters with respect to time, in particular the very fine time step does not influence the accuracy. The calculations have been performed for spatial boxes of different sizes. Since a few photoelectrons may be able to leave the cluster without being trapped by the Coulomb potential of the ionized cluster, they will move tens to a few hundreds of Angstroms during the time interval of the simulation. Employing a fixed spatial step size of $0.08 \AA$ in agreement with the corresponding de Broglie wave length, we started with a box size of $10 \AA$ with the cluster center of mass at the center of the box. The size of the box was increased by $1.0 \AA$ step by step, and the convergence of the calculated properties (distances, velocities, energies) was monitored up to $18 \AA$. Between the last two calculations, 17 and $18 \AA$, no visible differences in the displayed properties could be observed, i.e. the change compared to the last step was less than $1 \%$ in all cases.

\section{Results and discussion}

Within sub-femtoseconds of the X-ray field application, the electrons start to absorb the laser energy. In the following, the electronic and nuclear dynamics are presented in terms of various physical properties as functions of time.

To find out which orbitals are most influenced by the strong laser field, e.g. in electron-hole creation, the overlap between the initial $\psi_{i}(t=0)$ and time-developed molecular orbitals $\psi_{i}(t),\left|\left\langle\psi_{i}(t) \mid \psi_{i}(0)\right\rangle\right|^{2}$, has to be followed. Fig. 2 graphically displays these overlaps as a function of time during the first two femtoseconds, in the field with an intensity of $10^{16} \mathrm{~W} \mathrm{~cm}^{-2}$. In this short time interval, the displacement of the nuclei from the starting position is smaller than a single spatial grid step ( $0.08 \AA$ ). Up to about $1 \mathrm{fs}$ the fastest rate of change belongs to the higher valence orbitals. This behavior changes after $1 \mathrm{fs}$

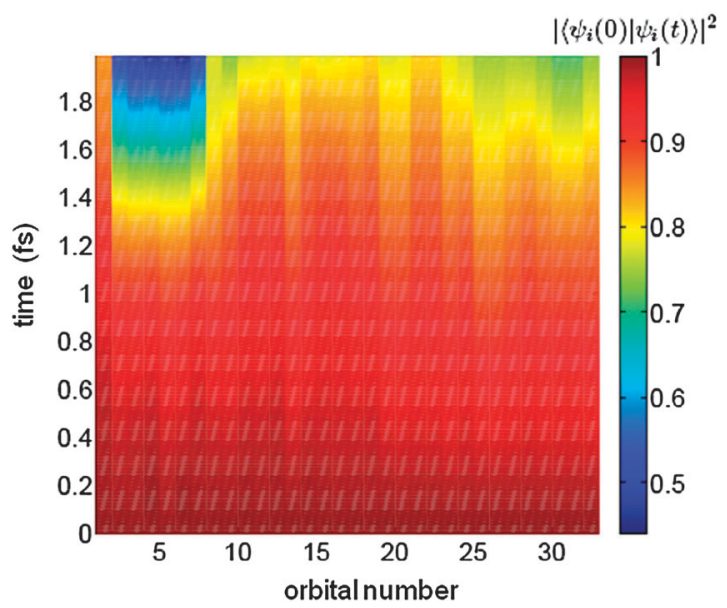

Fig. 2 The overlap between initial and time-developed molecular orbitals, under a laser field intensity of $10^{16} \mathrm{~W} \mathrm{~cm}^{-2}$. Orbitals are ordered by energy, from core (oxygen $2 \mathrm{~s}$ ) to valence orbitals, so that orbital no. 32 is the highest occupied molecular orbital. with a rapid drop of the overlap integral value for the oxygen $2 \mathrm{~s}$ orbitals, representing creation of electron holes in the corresponding orbitals. Due to symmetry there are two " $2 \mathrm{~s}$ " molecular orbitals (or rather two molecular orbitals formed from the eight oxygen $2 \mathrm{~s}$ atomic orbitals) which do not participate in the ionization process for the given polarization of the X-ray field (orbital nos. 1 and 8 in Fig. 2).

Fig. 3 shows the kinetic energy of the ions in the water cluster as a function of time. In Fig. 3(A) the kinetic energy corresponding to the two lower laser field intensities $10^{13} \mathrm{~W} \mathrm{~cm}^{-2}$ and $10^{14} \mathrm{~W} \mathrm{~cm}^{-2}$ is given, as both fall into the same scale. In the field of $10^{15} \mathrm{~W} \mathrm{~cm}^{-2}$ (Fig. 3(B)), the kinetic energy shows the first signs of changes in behavior. It no longer falls into the same scale as the kinetic energies of the two
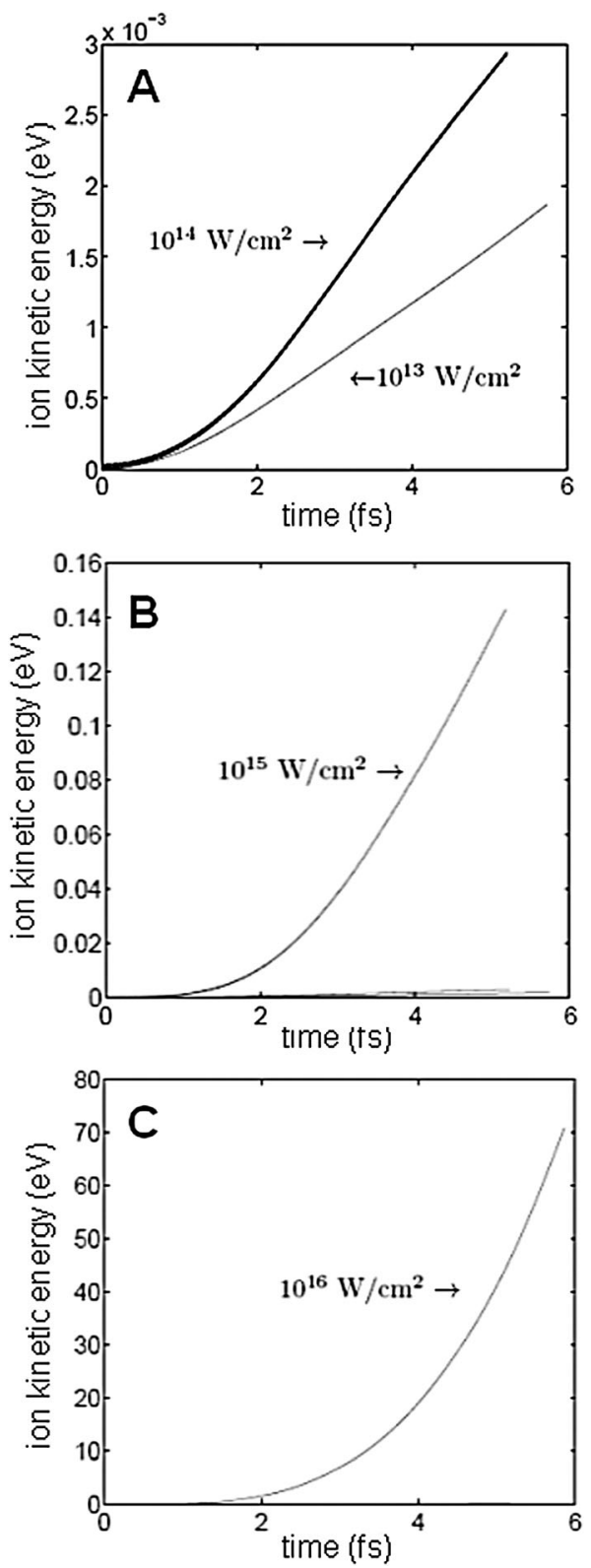

Fig. 3 Kinetic energy of the water cluster $\left(\mathrm{H}_{2} \mathrm{O}\right)_{8}$ under laser field intensities of (A) $10^{13} \mathrm{~W} \mathrm{~cm}^{-2}$ and $10^{14} \mathrm{~W} \mathrm{~cm}^{-2}$, (B) $10^{15} \mathrm{~W} \mathrm{~cm}^{-2}$, and (C) $10^{16} \mathrm{~W} \mathrm{~cm}^{-2}$. 

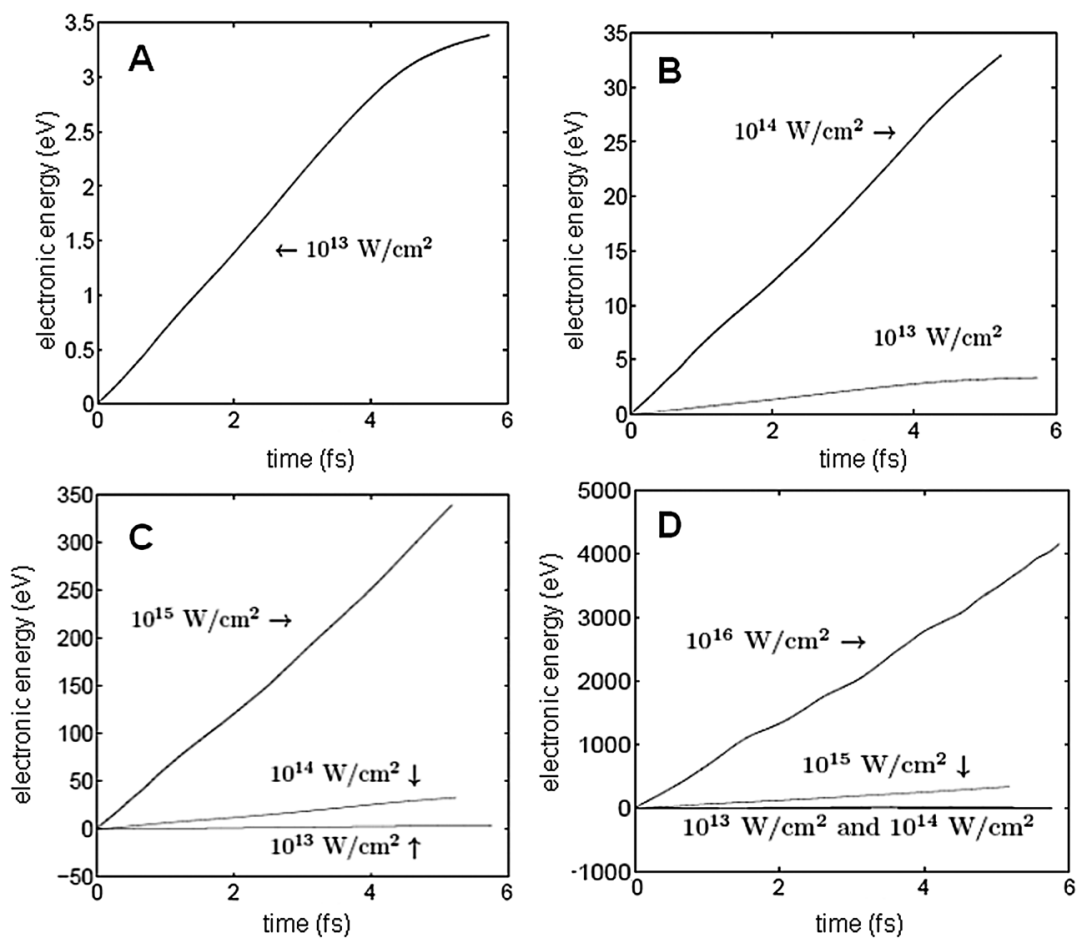

Fig. 4 Electronic energy of the water cluster $\left(\mathrm{H}_{2} \mathrm{O}\right)_{8}$ under laser field intensities of (A) $10^{13} \mathrm{~W} \mathrm{~cm}^{-2}$, (B) $10^{14} \mathrm{~W} \mathrm{~cm}^{-2},(\mathrm{C}) 10^{15} \mathrm{~W} \mathrm{~cm}^{-2}$ and (D) $10^{16} \mathrm{~W} \mathrm{~cm}^{-2}$.

lower fields, but it is up to two orders of magnitude higher at the end of the studied time interval. The influence that the strongest field has on the kinetic energy of the ions immediately becomes obvious from Fig. 3(C). Although this figure contains the kinetic energies corresponding to all four field strengths, only the one for the intensity $10^{16} \mathrm{~W} \mathrm{~cm}^{-2}$ can be seen, due to the fact that it is almost two orders of magnitude larger than even the kinetic energy corresponding to the field of intensity $10^{15} \mathrm{~W} \mathrm{~cm} \mathrm{~cm}^{-2}$. While for the weakest field the kinetic energy shows an almost linear dependence on time, this behaviour significantly changes for the stronger fields.

The main part of the energy contribution to the total energy increase (see Fig. 5) comes from the electronic energy (Fig. 4). In contrast to the kinetic energy increase, this increase is seemingly directly proportional to the field strength; averaged over the first 5 femtoseconds, $6 \times 10^{2} \mathrm{eV} \mathrm{fs}^{-1}$ for the strongest field, and about $0.6 \mathrm{eV} \mathrm{fs}^{-1}$ for the weakest. Note that the increase in the electronic energy with time is not strictly linear, but shows very slight oscillations. In general, the energy first has to be absorbed by the electrons before it can be transferred to ion kinetic energy and cause the Coulomb explosion.

The effect of the field strength on the ion movement can also be observed in terms of the direct ion response to the laser field oscillation. The thickness of the curve belonging to the field of $10^{14} \mathrm{~W} \mathrm{~cm}^{-2}$ in Fig. 3(A) is due to the response of the ions to the strong field, small oscillations corresponding to the laser field frequency. The different field strengths in these three cases are translated into the amplitude of the ion vibrations. For the strongest field, the kinetic energy oscillation amplitude amounts to about $4 \times 10^{-3} \mathrm{eV}$, which, for comparison, corresponds to about $T=50 \mathrm{~K}$ thermal energy. Over the whole simulation time-interval of $6 \mathrm{fs}$, the oscillation amplitude is larger than the total kinetic energy response of the two weaker laser fields. In the case of the weaker field $10^{14} \mathrm{~W} \mathrm{~cm}^{-2}$, the kinetic energy oscillation amplitude amounts to about $4 \times 10^{-5} \mathrm{eV}$ (corresponding to $T=0.5 \mathrm{~K}$ ), while for the field $10^{13} \mathrm{~W} \mathrm{~cm}^{-2}$ it is only $4 \times 10^{-6} \mathrm{eV}$ (or $T=0.05 \mathrm{~K}$ ).

The time-dependent differences of the atomic distances with respect to their initial positions (see Fig. 6) show an interesting behavior. As can be seen from Fig. 1, the water octamer exhibits $S_{4}$ symmetry, thus the hydrogen atoms can be organized in groups of four, depending on their position with respect to the center of the cluster. The time-dependent development of the atomic distances and velocities is in agreement with this cluster symmetry. The distance plots show a number of separate bunches of curves that correspond to symmetrically equivalent atoms. Most notable are the four outer hydrogen atoms, labeled $H(1)$, with the largest distance to the center of the cluster. From the point of view of velocities and displacements, this set of hydrogen atoms is in all four fields either among the fastest moving groups (for $10^{15} \mathrm{~W} \mathrm{~cm}^{-2}$ in Fig. 7(A)) or even the fastest moving group (in the other three cases) and so achieves the highest or the second highest displacement. On the opposite side the slowest, least moving hydrogens $\mathrm{H}(2)$ are found, which share the same water molecule with the fastest hydrogens $H(1)$. Note that for the two lower field strenghts, the overall distance range in the time interval under study is very similar; for $10^{-14} \mathrm{~W} \mathrm{~cm}$ cm $^{-2}$ intensity, there is no discernible difference in the distance change for both classes of oxygen atoms which, however, is rather accidental.

Although the total difference in the distance for the two weaker laser fields stays, in both cases, in the same order of magnitude, they show different time-dependent structural changes. Even the second strongest field causes only slight atomic displacements, 

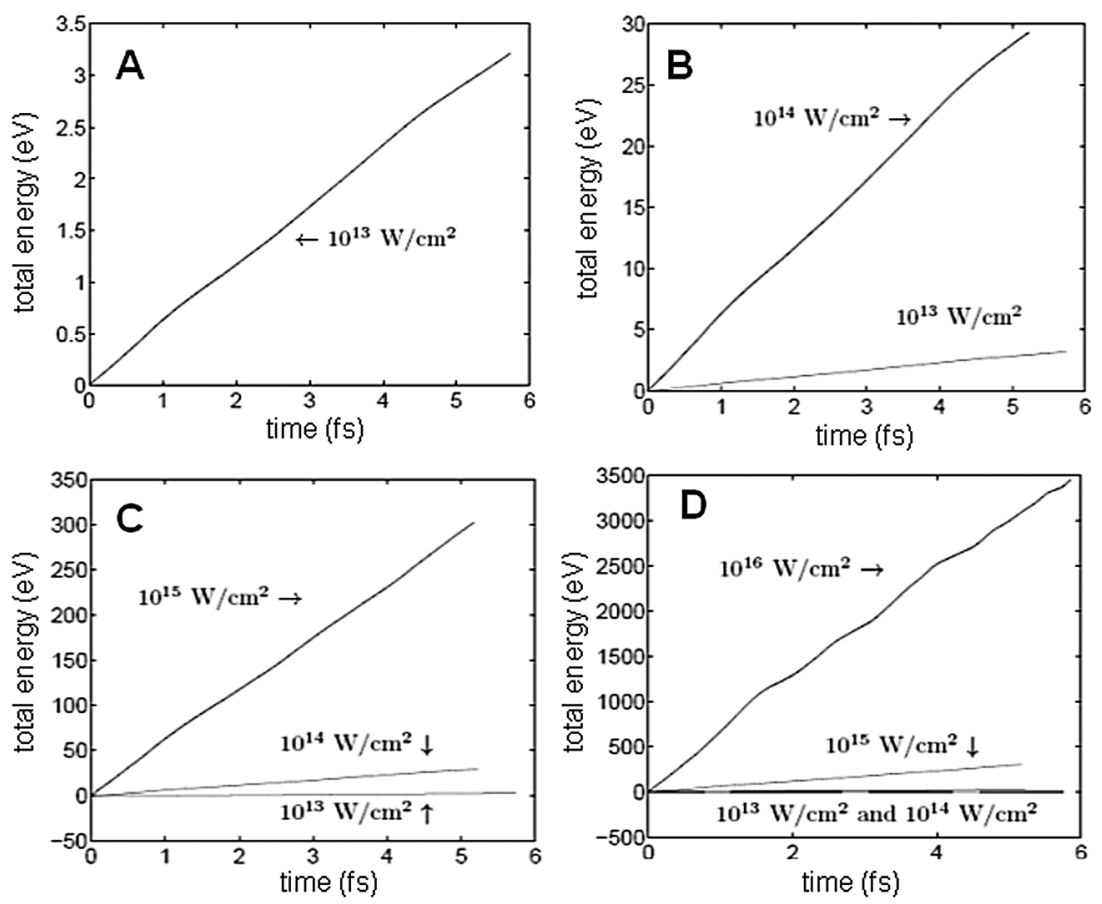

Fig. 5 Total energy of the water cluster $\left(\mathrm{H}_{2} \mathrm{O}\right)_{8}$ under laser field intensities of $(\mathrm{A}) 10^{13} \mathrm{~W} \mathrm{~cm}^{-2}$, (B) $10^{14} \mathrm{~W} \mathrm{~cm}^{-2}$, (C) $10^{15} \mathrm{~W} \mathrm{~cm}{ }^{-2}$ and $(\mathrm{D}) 10^{16} \mathrm{~W} \mathrm{~cm}^{-2}$.
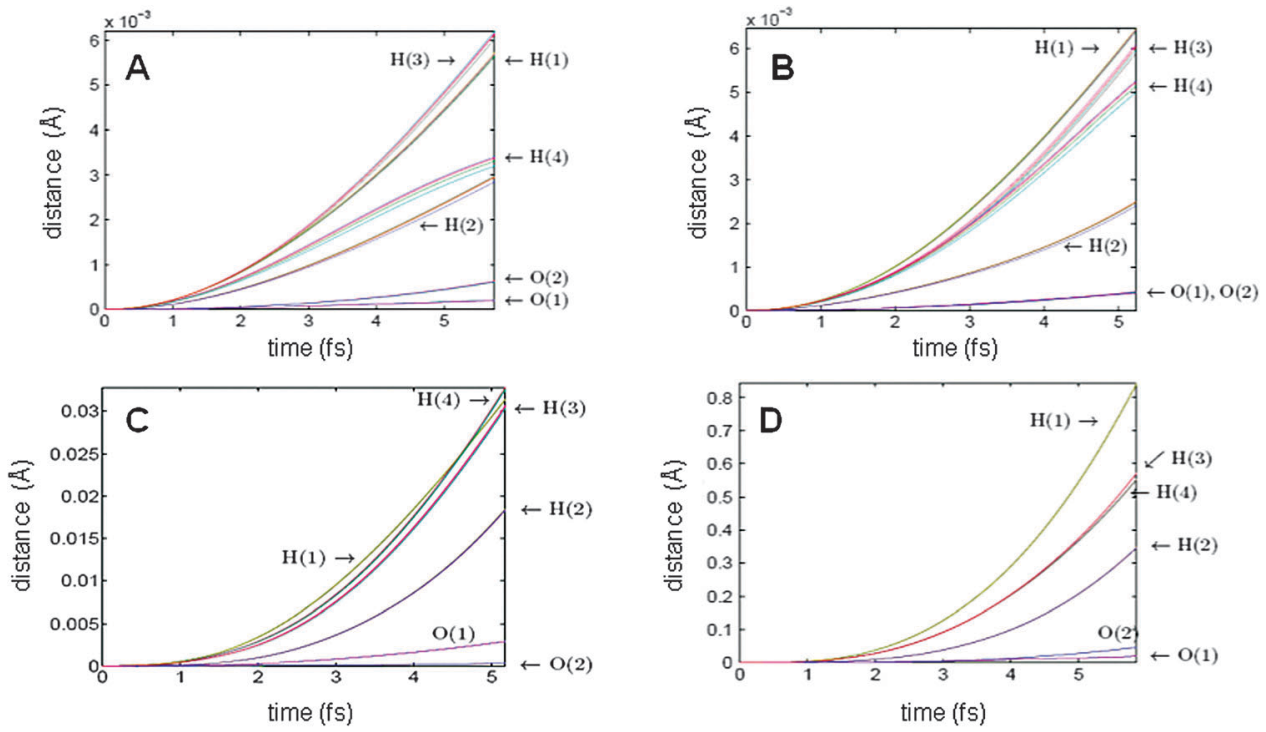

Fig. 6 Time evolution of the atomic distances from their initial positions at intensities of (A) $10^{13} \mathrm{~W} \mathrm{~cm}^{-2}$, (B) $10^{14} \mathrm{~W} \mathrm{~cm}{ }^{-2},(\mathrm{C}) 10^{15} \mathrm{~W} \mathrm{~cm}{ }^{-2}$ and (D) $10^{16} \mathrm{~W} \mathrm{~cm}^{-2}$. Several distinct groups of atoms with similar velocities form during the process. The atomic labels have been introduced in Fig. 1 .

which even at the end of the simulation time-interval are smaller than $0.08 \AA$, the size of the electron density spatial grid step. In all three cases of the weaker fields (Fig. 7(A)-(C)), the velocities for some of the atomic groups start to decrease after some times. Only the strongest field shows fast fragmentation corresponding to Coulomb explosion with the distance change of the outermost hydrogen atoms reaching $0.85 \AA$ in the first six femtoseconds. In this case, there is not any hint of a velocity drop over the whole simulation time-interval.

To compare the overall effect of the different field strengths, we estimate the displacements after times longer than the simulation interval using the velocities from the last stages of the computation. The results are quoted in Table 2 . We can see that all the three weaker fields cause only small displacements even after a relatively long time of $100 \mathrm{fs}$, while the strong field is responsible for a very fast movement of hydrogen atoms ( 2.8 to $5.7 \AA$ in $10 \mathrm{fs}$ ). Here, it should be stressed that only the strongest field exhibits the behavior of a Coulomb explosion as it could be inferred from the properties that have been presented so far. At the same time, as expected due to their mass, the movement of oxygen atoms is relatively slow even in the strong field where in the first $25 \mathrm{fs}$ we estimate a maximum displacement of only $0.36 \AA$. As the structural measurement is focused on heavier element atoms and not 

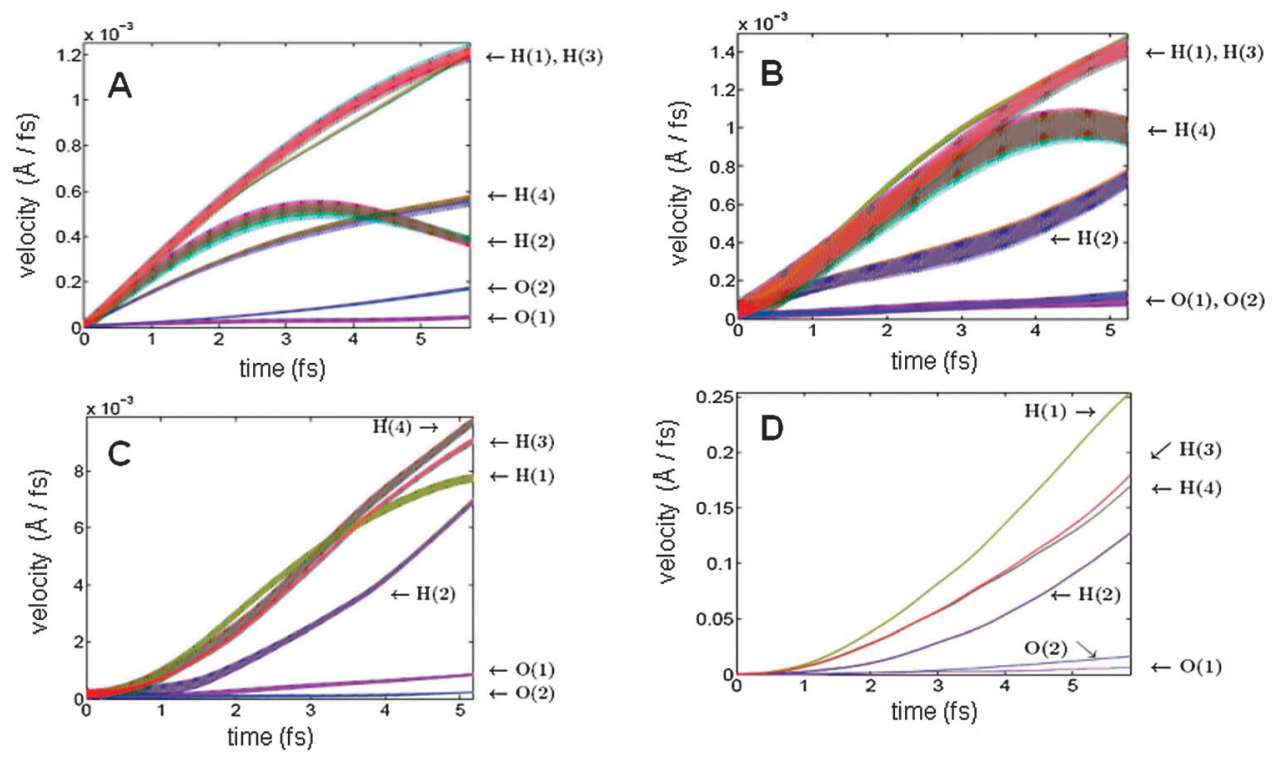

Fig. 7 Time evolution of the atomic velocities at intensities of (A) $10^{13} \mathrm{~W} \mathrm{~cm}^{-2}$, (B) $10^{14} \mathrm{~W} \mathrm{~cm}^{-2}$, (C) $10^{15} \mathrm{~W} \mathrm{~cm}^{-2}$ and (D) $10^{16} \mathrm{~W} \mathrm{~cm}^{-2}$, with atomic group labels as in Fig. 1. The fast oscillations correspond to the ion oscillations driven by the X-ray field.

Table 2 Estimated displacements based on the final velocities in the simulation

\begin{tabular}{|c|c|c|c|c|c|}
\hline \multirow{2}{*}{\multicolumn{2}{|c|}{$\frac{\text { Field }}{\mathrm{W} \mathrm{cm}^{-2}}$}} & \multicolumn{4}{|c|}{ Displacement (in $\AA$ ) after } \\
\hline & & $10 \mathrm{fs}$ & $25 \mathrm{fs}$ & $50 \mathrm{fs}$ & $100 \mathrm{fs}$ \\
\hline \multirow[t]{2}{*}{$10^{13}$} & $\mathrm{O}$ & $0.0004-0.0014$ & $0.001-0.004$ & $0.002-0.009$ & $0.004-0.017$ \\
\hline & $\mathrm{H}$ & $0.005-0.011$ & $0.011-0.030$ & $0.020-0.061$ & $0.038-0.122$ \\
\hline \multirow[t]{2}{*}{$10^{14}$} & $\mathrm{O}$ & $0.0007-0.0011$ & $0.0017-0.0034$ & $0.0034-0.0072$ & $0.007-0.015$ \\
\hline & $\mathrm{H}$ & $0.006-0.014$ & $0.016-0.036$ & $0.034-0.073$ & $0.069-0.148$ \\
\hline \multirow[t]{2}{*}{$10^{15}$} & $\mathrm{O}$ & $0.0014-0.0072$ & $0.0047-0.021$ & $0.01-0.043$ & $0.021-0.087$ \\
\hline & $\mathrm{H}$ & $0.051-0.080$ & $0.154-0.228$ & $0.325-0.474$ & $0.667-0.965$ \\
\hline \multirow[t]{2}{*}{$10^{16}$} & $\mathrm{O}$ & $0.046-0.12$ & $0.14-0.37$ & $0.31-0.78$ & $0.63-1.6$ \\
\hline & $\mathrm{H}$ & $0.87-1.9$ & $2.8-5.7$ & $6.0-12.1$ & $12.3-24.8$ \\
\hline
\end{tabular}

hydrogen, even pulses of this intensity and a length of up to 25 fs can lead to relatively good results. However, longer timescales of 50 to $100 \mathrm{fs}$ affect even the heavier oxygen atoms causing displacements of 0.8 to $1.6 \AA$.

Our study on the Coulomb explosion of the octamer water cluster provides a quantum-chemical rationalization of soft $\mathrm{X}$-ray FEL-matter interaction on chemical systems under real conditions. The chosen cluster is one of the dominant structures in bulk water. Since almost a century, the consistency of liquid water is a topic of structural studies. Besides the two phase model (ref. 24 and references therein) it is suggested that in liquid water tetrameric, octameric and dodecameric forms of water exist. ${ }^{25}$ Both models have been proved by complementary methods such as X-ray scattering (ref. 24 and references therein) and optical and X-ray spectroscopy. ${ }^{26,27}$

The octamer water cluster has been thoroughly investigated by time-independent quantum-chemical calculations. ${ }^{28-30}$ The water cubes with $D_{2 \mathrm{~d}}$ und $S_{4}$ symmetry have almost isoenergetical minima. ${ }^{31}$ Maeda and Ohno ${ }^{32}$ performed MP2//B3LYP calculations and found 164 minima on the potential energy surface with the $D_{2 \mathrm{~d}}$ structure being the global minimum, while the $S_{4}$-symmetric structure is more highly populated. There are two types of water monomers, donor-donor-acceptor and acceptor-donor-donor molecules. It should be noted that there exist cyclic and bicyclic octamer water clusters, ${ }^{33}$ which have less hydrogen bonds than the cubic forms and are thus energetically less favored. However, they are more stable due to entropic factors; free rotations around the hydrogen bonds are possible resulting in vibrational modes with low frequencies, while in the cubic forms the corresponding modes have considerably higher frequencies.

In our work on clusters we have chosen the $S_{4}$-symmetric $\left(\mathrm{H}_{2} \mathrm{O}\right)_{8}$ water cluster due to several reasons. It is of increased complexity in comparison to the well-structured tetrameric case without loosing its highly symmetric cubic structure. It is small enough to allow for a feasible calculation, and last but not least its geometry is very compact and can thus be compared with the nearly spherical cluster studied by Bergh, Timneanu and van der Spoel. ${ }^{7}$ These authors have used an electron-gas model to model the Coulomb explosion of a large water cluster. Their main finding was that the hydrogens move much faster than the oxygen atoms with the result that two shells of atomic cores are formed, the inner oxygen and an outer hydrogen shell. Obviously, our quantum-chemical studies show a very similar effect though not so pronounced (Fig. 8). Our study is much more detailed as we go beyond the free electron-gas model, including electron-correlation in the framework of the Kohn-Sham formalism. In particular, we can study the dynamics of the Coulomb explosion with respect to electron orbitals (also within the Kohn-Sham picture).

Both octamers have been detected by Buck and co-workers, ${ }^{34}$ making use of a combination of a size-selection of the clusters (by momentum transfer in encounters with rare-gas atoms) and the IR spectroscopic technique. Thus, it should be possible that the Coulomb explosion of the octamer water cluster can be investigated experimentally and the measurements can be compared with the results of the present work.

Finally, the time-dependent density functionals used for describing the behavior of the electrons in strong fields are 


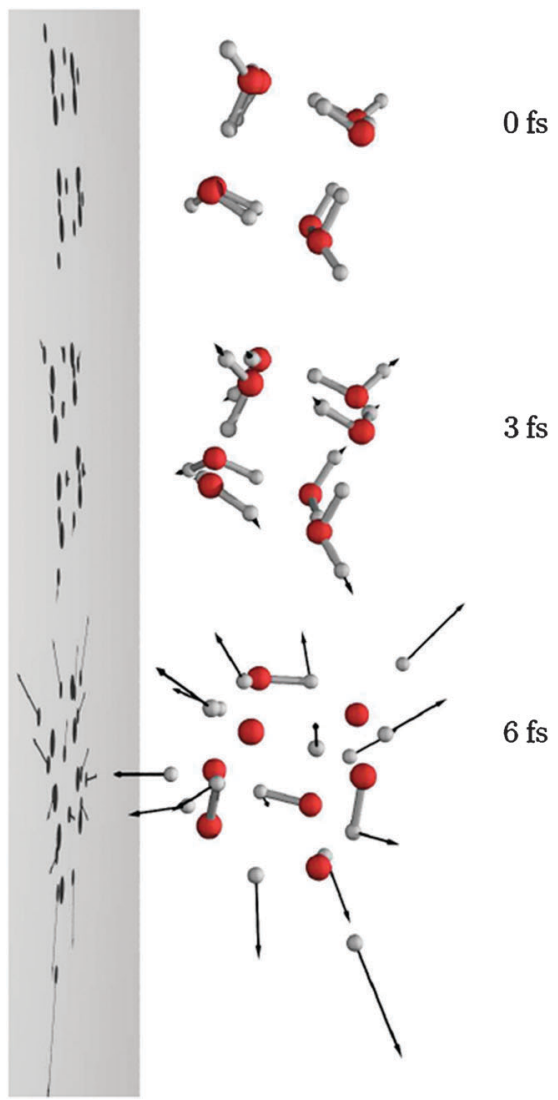

Fig. 8 The Coulomb explosion of the water cluster $\left(\mathrm{H}_{2} \mathrm{O}\right)_{8}$ in the field of $10^{16} \mathrm{~W} \mathrm{~cm}^{-2}$ with velocity vectors. Shown are the situations for $t=0 \mathrm{~s}, 3 \mathrm{~s}$ and $6 \mathrm{~s}$.

still in a developmental stage. Thus, time-dependent scattering measurements, in particular on Coulomb explosions, are a valuable tool for obtaining a better understanding of the general behavior of electrons in strong fields and contribute to an improvement in the precision of TD density functionals.

\section{Conclusions}

Theoretical studies of X-ray laser-matter interaction have gained importance in recent years in connection with proposed single-molecule coherent diffraction measurements. These measurements are intended for relatively large molecular systems, especially various bio-molecules for which conventional crystallography is not an option. However, the theoretical treatment of the interaction between large molecular systems and X-ray lasers necessarily employs severe approximations in order to decrease the computational costs. To describe the X-ray laser-matter interaction in a quantummechanical approach we have limited the size of the studied system to a small water cluster, $\left(\mathrm{H}_{2} \mathrm{O}\right)_{8}$. In the current implementation in the OCTOPUS program package the external laser pulse field cannot treat hard X-ray energies, due to the dipole approximation and the description of atomic core electrons through pseudo potentials. In principle, it is possible to overcome these limitations and to extend the method into the hard X-ray regime.
TDDFT may become an important tool for estimating which field strengths and pulse lengths are necessary to obtain a scattering signal of acceptable quality. A detailed quantummechanical analysis of the X-ray laser-matter interaction in small molecular systems can play an important role in choosing optimal field parameters for structural measurements. However, the limitations that current density functionals impose should be kept in mind. The time-dependent electron density measurement can provide a motivation for the development of new density functionals, as an additional means of comparison of TDDFT performance with real electron wave function behavior.

In this work, we made use of one of the main advantages of the TDDFT approach, that the detailed quantum-chemical electron density of the system can be followed in time as it changes under the influence of the external field. As shown, the occupation numbers of the molecular orbitals can be studied as functions of time. No assumptions have to be made about the nature of the excitation and ionization processes. This is particularly important for strong fields where nonlinear effects cannot be neglected, exemplified by the valence excitations evident from Fig. 2.

The strongest field considered in our simulation $\left(10^{16} \mathrm{~W} \mathrm{~cm}^{-2}\right)$ leads to very fast cluster fragmentation, which renders a structural measurement meaningless already after a few femtoseconds. As has been shown, the scattering intensity on the main oxygen maxima drops by $12 \%$ within just 5 femtoseconds. The Coulomb explosion sets in very fast with an electron plasma formation, the scattering signal of which contributes to the diffuse part of the scattering spectrum. Oxygen ions move relatively slowly even in the strongest field preserving the overall structure of the cluster.

Depending on the objectives of the experiment, an encouraging result is the relatively well-conserved overall symmetry and structure on short time-scales. This is good news if one is interested in understanding the overall structure of the sample. However, the possibility to determine any details within the structure will be lost very quickly due to the fast plasma formation. As we could show, in particular for the heavier atoms the scattering intensity is very sensitive to the electron plasma build-up although the actual ion movements during the first stages of the Coulomb explosion are negligible. Thus, it is important that simulations of Coulomb explosions accurately describe the detailed changes of the electron density.

\section{Acknowledgements}

This work was supported by DFG/SFB755 Nanoscale Photonic Imaging. S.T. is grateful to the Aventis Foundation and A.D. thanks SFB755 for additional financial help. S.T. thanks the Advanced Study Group of the Max Planck Society for continuous support. S.S. and S.T. are grateful for generous financial support by the Fonds der Chemischen Industrie.

\section{References}

1 U. Saalmann, C. Siedschlag and J. M. Rost, J. Phys. B, 2006, 39, R39.

2 K. Yamanouchi, Science, 2002, 295, 1659.

3 S. P. Hau-Riege, R. A. London and A. Soke, Phys. Rev. E: Stat. Phys., Plasmas, Fluids, Relat. Interdiscip. Top., 2004, 69, 051906.

4 Z. Jurek, G. Faigel and M. Tegze, Eur. Phys. J. D, 2004, 29, 217. 
5 Z. Jurek and G. Faigel, Europhys. Lett., 2004, 65, 491.

6 R. Neutze, R. Wouts, D. van der Spoel, E. Weckert and J. Hajdu, Nature, 2000, 406, 752.

7 M. Bergh, N. Timneanu and D. van der Spoel, Phys. Rev. E: Stat. Phys., Plasmas, Fluids, Relat. Interdiscip. Top., 2004, 70, 051904.

8 S. P. Hau-Riege, R. A. London, H. N. Chapman and M. Bergh, Phys. Rev. E: Stat. Phys., Plasmas, Fluids, Relat. Interdiscip. Top., 2007, 76, 046403.

9 S. Karthikeyan, M. Park, I. Shin and K. S. Kim, J. Phys. Chem. A, 2008, 112, 10120

10 C. J. Gruenloh, J. R. Carney, C. A. Arrington, T. S. Zwier, S. Y. Fredericks and K. D. Jordan, Science, 1997, 276, 1678.

11 Q. Du, E. Freysz and Y. Shen, Phys. Rev. Lett., 1993, 70, 2313.

12 B. Rowland, N. Kadagathur, J. Devlin, V. Buch, T. Feldman and M. Wojcik, J. Chem. Phys., 1995, 102, 8328.

13 A. Castro, H. Appel, M. Oliveira, A. Rozzi, X. Andrade, F. Lorenzen, M. A. L. Marques, E. K. U. Gross and A. Rubio, Phys. Status Solidi B, 2006, 243, 2465.

14 M. Marques, A. Castro, G. F. Bertsch and A. Rubio, Comput. Phys. Commun., 2003, 151, 60.

15 M. Isla and J. A. Alonso, Phys. Rev. A, 2005, 72, 023201.

16 E. Livshits and R. Baer, J. Phys. Chem., 2006, 110, 8443.

17 R. Baer and D. Neuhauser, Phys. Rev. Lett., 2005, 94, 043002.

18 T. Ziegler, M. Seth, M. Krykunov and J. Autschbach, J. Chem. Phys., 2008, 129, 184114.

19 B. X. Yang and J. Kirz, Phys. Rev. B: Condens. Matter Mater. Phys., 1987, 36, 1361.
20 H. Hayashi, N. Watanabe, Y. Udagawa and C. Kao, Proc. Natl. Acad. Sci. U. S. A., 2000, 97, 6264.

21 W. J. Veigele, At. Data, 1973, 5, 51.

22 N. Timneanu, C. Caleman, J. Hajdu and D. van der Spoel, Chem. Phys., 2004, 299, 277.

23 D. J. Wales, J. P. K. Doye, A. Dullweber, M. P. Hodges, F. Y. Naumkin, F. Calvo, F. J. Hernandez-Rojas and T. F. Middleton, The Cambridge Cluster Database., 2011, http://www-wales.ch.cam. ac.uk/CCD.html.

24 A. S. F. Ramos and S. Techert, Biophys. J., 2005, 89, 1990.

25 R. Ludwig, Angew. Chem., Int. Ed., 2001, 40, 1808.

26 L.-A. Näslund, D. C. Edwards, P. Wernet, U. Bergmann, H. Ogasawara, L. G. M. Pettersson, S. Myneni and A. Nilsson, J. Phys. Chem. A, 2005, 109, 5995.

27 U. Bergmann, A. Di Cicco, P. Wernet, E. Principi, P. Glatzel and A. Nilsson, J. Chem. Phys., 2007, 127, 174504.

28 J. Kim, B. J. Mhin, S. J. Lee and K. S. Kim, Chem. Phys. Lett., 1994, 219, 243.

29 J. Kim, D. Majumdar, H. M. Lee and K. S. Kim, J. Chem. Phys., 1999, 110, 9128.

30 S. D. Belair and J. S. Francisco, Phys. Rev. A, 2003, 67, 063206.

31 F. Weinhold, J. Chem. Phys., 1998, 109, 367.

32 S. Maeda and K. Ohno, J. Phys. Chem. A, 2007, 111, 4527.

33 F. Weinhold, J. Chem. Phys., 1998, 109, 373.

34 U. Buck, I. Ettischer, M. Melzer, V. Buch and J. Sadley, Phys. Rev. Lett., 1998, 80, 2578. 